\title{
Optimizing optics and opto- mechanical mounting to minimize static aberrations in high-contrast instruments
}

D. Echeverri, N. Jovanovic, J.-R. Delorme, G. Ruane, J. Fucik, et al.

D. Echeverri, N. Jovanovic, J.-R. Delorme, G. Ruane, J. Fucik, J. K. Wallace, D. Mawet, "Optimizing optics and opto-mechanical mounting to minimize static aberrations in high-contrast instruments," Proc. SPIE 10703, Adaptive Optics Systems VI, 1070358 (13 July 2018); doi: 10.1117/12.2313887

Event: SPIE Astronomical Telescopes + Instrumentation, 2018, Austin, Texas, United States 


\title{
Optimizing optics and opto-mechanical mounting to minimize static aberrations in high-contrast instruments
}

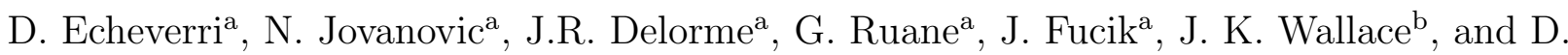 \\ Mawet $^{\mathrm{a}, \mathrm{b}}$ \\ aDepartment of Astronomy, \\ California Institute of Technology, Pasadena, CA 91106, USA \\ ${ }^{\mathrm{b}}$ Jet Propulsion Laboratory, \\ California Institute of Technology, Pasadena, CA 91109, USA
}

\begin{abstract}
One of the goals of high-contrast imaging is to reach contrasts of $10^{-10}$ at small inner working angles to directly image Earth-like exoplanets around solar-type stars. In most imaging systems, a deformable mirror (DM) in the pupil plane can correct for phase errors but surface errors in out-of-pupil optics get coupled into amplitude errors which can only be controlled with a second, out of pupil, DM. Furthermore, correcting static errors introduced by the optics can take up valuable DM stroke. Thus, minimizing the wavefront error within the system is critical to reaching high contrast levels. For example, the High Contrast Spectroscopy for Segmented Telescopes Testbed (HCST) in the Exoplanet Technologoy lab at Caltech aims to develop exoplanet imaging technologies down to small inner working angles $(<3 \lambda / D)$ which requires an RMS wavefront error of less than $10 \mathrm{~nm}$ per optic to achieve a contrast of $10^{-5}$ with the DM flattened. While aligning HCST, it was determined that despite the excellent surface quality of all the optics, the mounts were introducing significant wavefront errors. Here we assess the effect of mount-induced wavefront errors that can rapidly consume the wavefront budget of a high-contrast system. We also present the method used to mitigate this effect within HCST such that a mean contrast of $6 \times 10^{-6}$ from $3-10 \lambda / D$ was achieved with a vortex coronagraph and flattened DM.
\end{abstract}

Keywords: High Contrast Imaging, Instrumentation, Opto-Mechanical Systems

\section{INTRODUCTION}

The goal of high-contrast imaging systems is to detect and characterize exoplanets by directly capturing their light. This is challenging because a planet's light is significantly dimmer than its host star's. For example, an Earth-like planet orbiting a Sun-like star at $1 \mathrm{AU}$ is $10^{10}$ times dimmer than its star and, as such, the planet light is overwhelmed by the diffracted starlight and scattering generated by wavefront aberrations. Various types of coronagraphs exist which can theoretically achieve contrasts better than $10^{-10}$ and contrasts of $3.2 \times 10^{-10}$ have been demonstrated in practice ${ }^{1,2}$ but they are still uncommon. The discrepancy in performance is primarily due to imperfections in the wavefront which undermine the coronagraph's ability to suppress starlight thereby allowing some of this light to pass and manifest as speckles in the focal plane. In a laboratory environment, the wavefront aberrations affecting coronagraph performance are due to multiple factors such as air turbulence, thermal drifts, vibrations, the surface quality of the optics, and so on. Surface quality includes not only the high spatial frequency effects like surface roughness but also low-order effects like the nominal shape of the optic.

Adaptive optics (AO) or wavefront control can be used to compensate for most of the effects described above. In general, a deformable mirror (DM) is placed in the pupil plane so that it can correct for phase errors present in the wavefront. Since it is in the pupil though, it can only introduce a phase shift on the wavefront which means that any amplitude errors cannot be controlled with that DM. This is sufficient for correcting many types of errors, including surface errors on optics that also reside in the pupil plane of the system. However, out-of-pupil optics introduce phase errors that evolve into amplitude as they propagate to the focal plane. A second DM can be placed out of the pupil so that it can compensate for these, and other, amplitude errors. However, this means

Send correspondence to dechever@caltech.edu 
that some of the stroke on both DMs is used to compensate for imperfections in the optics themselves instead of correcting for dynamic environmental factors such as thermal drifts and air turbulence. Ideally the full stroke of the DM is reserved for errors that can't be controlled through careful system design and alignment.

To minimize static aberrations introduced by the optics in the system, their flatness and material properties are often specified carefully during the design and build of a high-contrast testbed. For example, each of the optics in the High Contrast Spectroscopy for Segmented Telescopes Testbed (HCST) at Caltech was specified to have $<10 \mathrm{~nm}$ RMS reflected wavefront error in the low spatial frequencies and less than $30 \mathrm{~nm}$ total peak-tovalley surface error over the entire optic. Simulations of the full system showed that these values were required to reach a contrast of $10^{-5}$ with the DMs flat so that common-path wavefront sensing techniques could then be used to dig dark holes far deeper than this without having to compensate for the optical quality of the system. Nevertheless, during the alignment of HCST, it was determined that the mounts were deforming the optics and introducing significant wavefront errors. As such, the optimization of the surface quality was lost to the mounting scheme and there were large static errors in the initial alignment. To correct these mount-induced aberrations, each mount used in the testbed was analyzed in isolation to determine its effect on the wavefront quality of the system. This process revealed that some mounting schemes had a larger effect than others. Other commercially available mounts were also analyzed for comparison and these results guided an alignment procedure in which each optic was tuned individually in front of an interferometer to minimize the mount-induced errors before being placed in the testbed. That procedure was used to successfully align two separate systems to high precision at Caltech. HCST, with 17 of its optics aligned, and the Fiber Injection Unit (FIU) for the Keck Planet Imager and Characterizer (KPIC), ${ }^{3}$ with all 8 reflective surfaces and 2 transmissive optics aligned, both had an RMS wavefront error of $<30 \mathrm{~nm}$ through the whole system.

\section{METHODS}

All the measurements and alignment for HCST and KPIC were done with a Zygo Verifire interferometer (Zygo). Since the Zygo outputs a collimated beam, it was relatively easy to analyze flat mirrors; each flat was placed in its mount and then set in front of the Zygo as shown in Fig. 1. The flat was then adjusted in angle to remove any tip and tilt since these aberrations are purely alignment-induced in a flat.

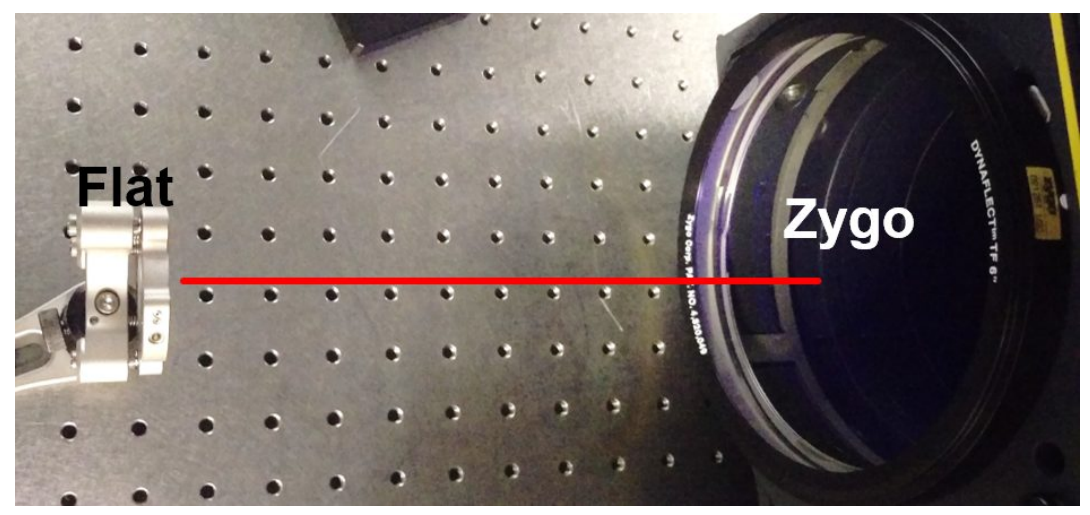

Figure 1. Setup for analyzing flat mirrors. Here a 1" flat is being tested with the Zygo interferometer, seen on the right.

A slightly different setup had to be used for off-axis parabolic (OAP) mirrors. OAPs were mounted and then aligned so that the Zygo's collimated beam was parallel to their parent axis. A high-quality, silicon nitride ball was placed at the focus and served as the reference for reflecting the light back into the Zygo. This setup is shown in Fig. 2. The reference ball was placed on a three-axis translation stage so that it could always meet the focus of the OAP and the OAP mounts had actuators for tip and tilt so the optic could be aligned with the incoming beam. Once the OAP and reference ball were aligned, the Random Ball Test ${ }^{4,5}$ was used to average out any surface defects on the ball. As stated in Ref. 4, using a ball for analyzing OAPs with a slow f\# leads to diffractive ringing which can be seen in all the OAP wavefront maps collected, eg. Fig. 7. However, this ringing was low-amplitude, high-frequency, and regular so it could easily be discerned from actual aberrations. 


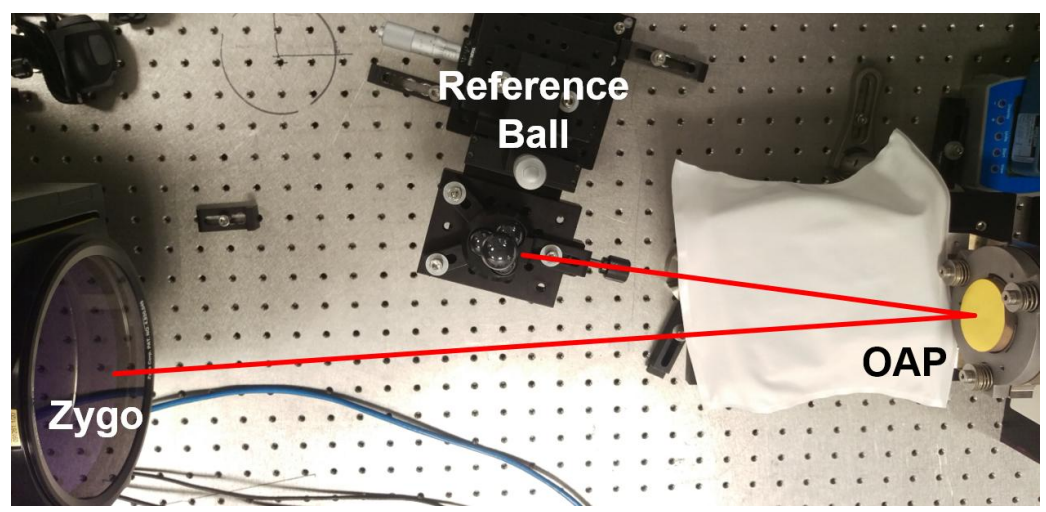

Figure 2. Setup for analyzing OAPs. The Zygo's output window is shown on the bottom left, the OAP is on the right, and the reference ball is centered at the top of the image. Note that the ball is on a 3-axis translation stage.

The five degrees of freedom in the OAP setup were key to measuring the wavefront but the order in which they were used had to be carefully considered. This is because it is easy to mask mount-induced aberrations with alignment errors. For example, one of the primary errors introduced by the mounts we tested was astigmatism. However, astigmatism can also be introduced in an OAP by tilting it such that the collimated beam is angled with respect to the parent axis. Thus, it is hard to tell whether the astigmatism present in a measurement is due to misalignment or to the mount. The following procedure was developed to ensure that mount-induced aberrations could be distinguished from alignment errors.

1. The OAP was mounted with the set screws backed off so that it was held in place by its own weight.

2. The wavefront error measured by the Zygo was minimized by angling the OAP and translating the ball. The final wavefront was recorded as the "nominal" value for the OAP as it was under no mounting stress.

3. The OAP was adjusted so that its back surface, which is perpendicular to the parent axis, was flush with the mount. It was assumed that this motion only introduced an angle since we were careful not to rotate the OAP. The OAP was held in this position while the next step was performed.

4. One of the set screws was tightened with a torque gauge until it just barely made contact with the OAP.

5. The OAP was angled so that the aberrations were again minimized but the reference ball was not moved since its position was defined by step 2; the OAP just needed to be tilted back into place so that its parent axis was aligned with the Zygo beam.

6. The torque on the set screws was gradually increased and the wavefront was recorded. Neither the OAP nor the ball were moved. This is because the set screw is centered and isolated from the OAP by a flexure arm so it cannot transfer any torque to the optic and any loads are compressive and radial. As such, the OAP's parent axis was unlikely to move and any aberrations were due to the mount deforming the optic.

Several optics and mounts were analyzed with these methods but only a few are presented here:

- A $50.0 \mathrm{~mm}$ diameter, $10 \mathrm{~mm}$ thick, custom Zerodur flat mirror from HCST.

- A $25.4 \mathrm{~mm}$ diameter, $7 \mathrm{~mm}$ thick, custom Zerodur flat mirror from HCST.

- A $50.0 \mathrm{~mm}$ diameter, $12.4 \mathrm{~mm}$ average thickness, custom Zerodur OAP from HCST. The off-axis distance is $50.3 \mathrm{~mm}$ and the effective focal length is $334.9 \mathrm{~mm}$.

The mounts presented are:

- A 50.8 mm Newport Suprema Clear Edge Mount (Model: SN200-F3K).

- A 50.8 mm Newport Gimbal Mount (Model: GM2).

- A $50.8 \mathrm{~mm}$ Custom OAP mount from HCST.

- A $25.4 \mathrm{~mm}$ Thorlabs Polaris Flexure Spring Mount (Model: POLARIS-K1). 
- A $25.4 \mathrm{~mm}$ Thorlabs Polaris Flexure Clamp Fixed Mount (Model: POLARIS-B1S).

- A $25.4 \mathrm{~mm}$ Newport Suprema Clear Edge Mount (Model: SN100-F3K).

\section{RESULTS}

\subsection{Mounts at Minimal Holding Torque}

The first test determined the wavefront error introduced when the set screw on each mount was torqued to the minimal level where the optic was securely held. The metric for "securely held" was when the mirror could be tapped lightly on the back without introducing significant misalignment on the Zygo. This is similar to a test performed by Thorlabs on their Polaris line of mounts except that they measured the force required to push an optic out of the mount. However, since high-contrast testbeds should be relatively stable, the push-out criterion would have been excessive for our tests and a tap test was settled on instead. The two flat mirrors were used for these experiments since they were simpler and faster to test; any wavefront error relative to the nominal measurement, other than tip and tilt, had to come from the mount itself, not alignment.

For the $50.0 \mathrm{~mm}$ flat, two commercially available, off-the-shelf (COTS) $50.8 \mathrm{~mm}$ mounts were analyzed: a Newport Suprema and a Newport Gimbal mount. The Suprema line is Newport's "flagship" series while the Gimbal mount is discontinued but still common. Neither mount had a flexure like the ones in the $25.4 \mathrm{~mm}$ mounts so the Gimbal mount was tested with soft and regular-tip set screws, while the Suprema was tested with the soft-tip screw it came with. This provided a balance of high-end and standard mounting schemes. Figure 3 shows the resulting wavefront maps along with a case where the set screw was completely backed-off as a reference. The four maps have the same color bar to highlight changes relative to the nominal.

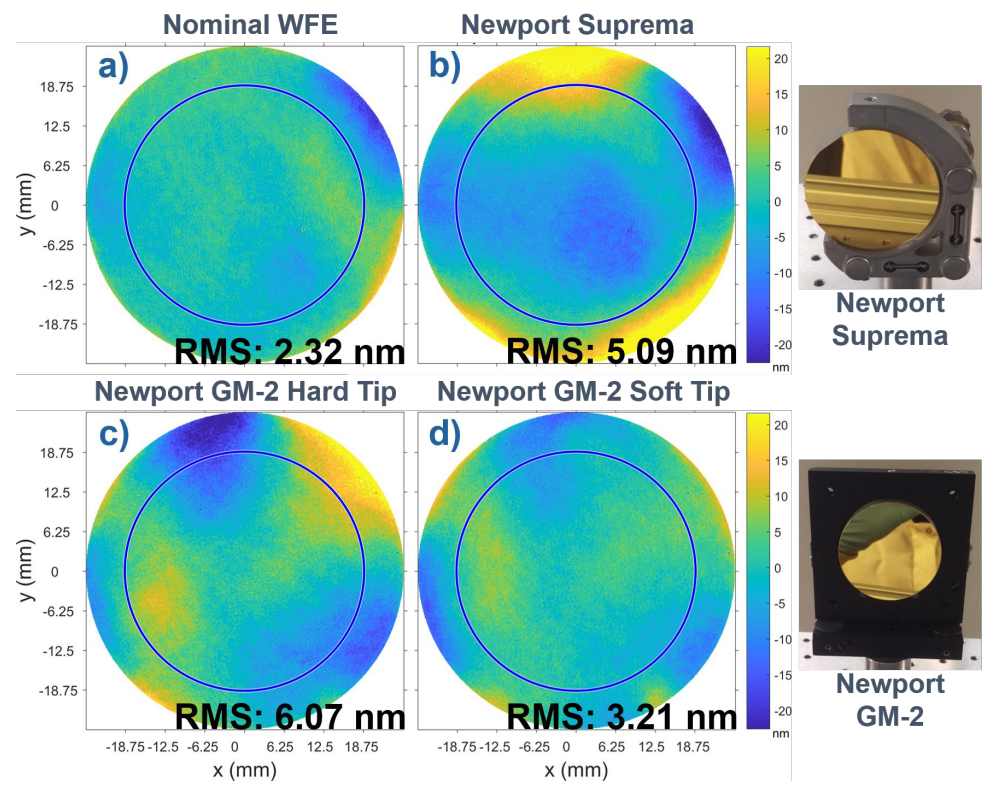

Figure 3. Wavefront maps for a $50.0 \mathrm{~mm}$ flat mirror at the minimal holding torque for each mounting scheme. The RMS value within an $80 \%$ region, indicated by the blue circle, is reported at the bottom of each image. a) is the nominal wavefront where the flat is not under any mounting stress, b-d) are the same mirror at the minimum mounting stress required to securely hold it in three different mounting schemes. The 2 mounts used are shown at the right of the figure.

As expected, all the mounts introduced some wavefront error. Most of it was concentrated around the edges of the mirror where the mount made contact with the optic but some error propagated into the $80 \%$ region denoted by the blue circle. From these results, it appears that the best mount is the GM- 2 with a soft set screw but it is important to note that this mount holds the mirror with 2 rubber pads so it may not be as stable as the higher-end Suprema. The Suprema is the one used in HCST and, as shown here, it doubles the RMS error relative to the nominal shape but it is still significantly under the requisite $10 \mathrm{~nm}$ RMS reflected wavefront error. 
The same experiment was repeated with the $25.4 \mathrm{~mm}$ flat mirror and three $25.4 \mathrm{~mm}$ COTS mounts. The mounts were: a Thorlabs Polaris Flexure Spring mount, a Thorlabs Polaris Flexure Clamp mount, and a Newport Suprema mount. The Polaris line of mounts is Thorlabs's high-end, ultra-stable series. The Flexure Spring mount has a thin leaf spring to reduce the stress transferred to the mirror while the Flexure Clamp mount has a much thicker clamping arm which, according to Thorlabs, provides better grip and stability while still minimizing the stress on the optic. The Suprema mount is the $25.4 \mathrm{~mm}$ analog to the $50.8 \mathrm{~mm}$ one tested before. Figure 4 shows the resulting wavefront maps for this test. As before, the maps have the same color bar.

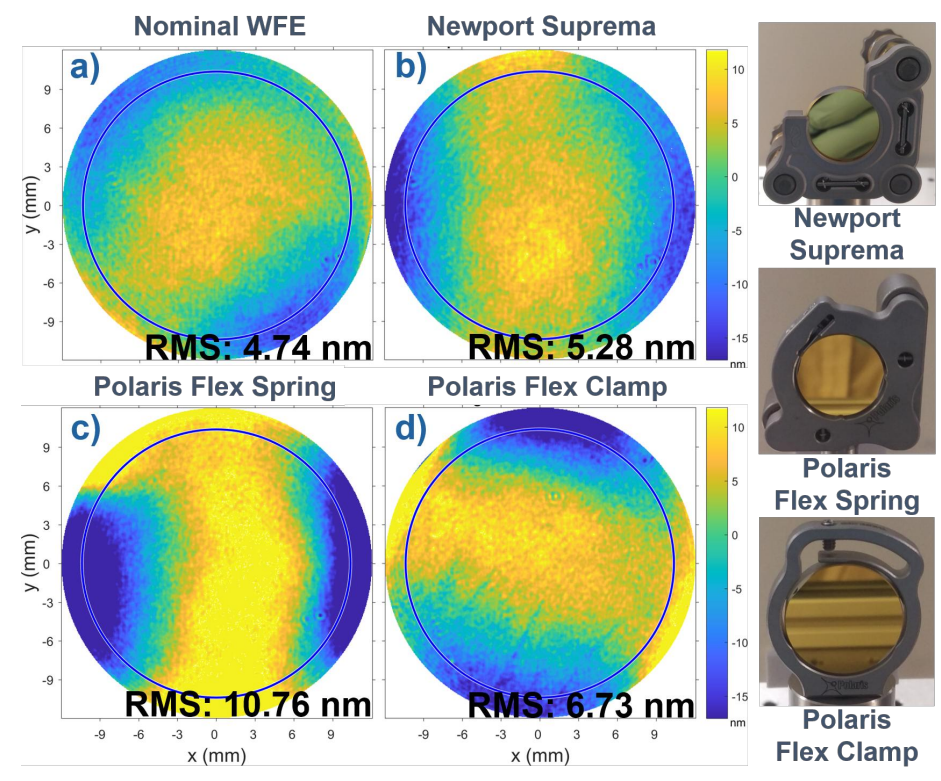

Figure 4. Wavefront and RMS of a $25.4 \mathrm{~mm}$ flat at the minimum holding torque in various mounts. a) is the nominal wavefront, b-d) have the minimum mounting stress required in the three mounts shown on the right.

Overall, every mount strained the flat mirrors and introduced wavefront errors but, looking at the RMS values, the deviation from the nominal was more pronounced in the $50.0 \mathrm{~mm}$ mirror. The only $25.4 \mathrm{~mm}$ mount to introduce significant wavefront error was the Polaris Flex Spring but this is likely due to the fact that its set screw had to be torqued more than the other $25.4 \mathrm{~mm}$ mounts in order to properly grip the mirror. This matches Thorlabs's statement that the Polaris Flex Clamp provides a better grip with less strain. However, as shown in Sec. 3.2, the rate at which the mirror deforms the optic must be considered as well and the Flex Clamp mount has a particularly steep response. As such, it takes significantly less torque to grip the mirror with this mount but it is also easier to over-tighten and accidentally introduce a great deal of wavefront error.

Table 1 summarizes all the key values for the various mounts at their minimum holding torque. The rows are arranged in order of increasing RMS wavefront error within the $80 \%$ central surface region. As stated before, all the mounts produced some wavefront error but only one of them, the Polaris Flex Spring, failed the $10 \mathrm{~nm}$ requirement for HCST. This is actually the mount used for most of the OAPs in the KPIC instrument but it is possible that the deformation seen in that instrument was less because of the fact that the OAPs were thicker than the $7 \mathrm{~mm}$ flat used in these tests. Table 1 also includes the RMS wavefront error from astigmatism. The effect of astigmatism on alignment is discussed in Sec. 3.3 but it is worth noting here that the contribution of astigmatism to the RMS error within the $80 \%$ region was significant for all the mounts despite the fact that they were at their minimal holding torque. As such, even after carefully tuning and minimizing the strain within any given optic, as described in Sec. 4, there will be slight alignment errors because of the mount-induced aberrations. This, along with the fact that the RMS error can double or triple in many of these mounts despite tuning, makes it clear that the mounts should also be given careful consideration when designing a high-contrast testbed, especially with larger optics. If the wrong mount is used, much of the wavefront optimization gained from purchasing high-quality optics could be lost to mounting stresses. 
Table 1. Summary of key values from the minimum hold tests.

\begin{tabular}{l|cccc}
\hline Mount & $\begin{array}{c}\text { RMS } \\
\text { Full Surface } \\
{[\mathrm{nm}]}\end{array}$ & $\begin{array}{c}\text { RMS } \\
80 \% \text { Surface } \\
{[\mathrm{nm}]}\end{array}$ & $\begin{array}{c}\text { RMS Astigmatism } \\
80 \% \text { Surface } \\
{[\mathrm{nm}]}\end{array}$ & $\begin{array}{c}\text { Torque on } \\
\text { Set Screw } \\
{[\text { oz-in] }}\end{array}$ \\
\hline $50.8 \mathrm{~mm}$ Nominal & 4.07 & 2.32 & 0.28 & N/A \\
$50.8 \mathrm{~mm}$ GM-2 Soft Screw & 4.66 & 3.21 & 1.09 & 3.0 \\
$50.8 \mathrm{~mm}$ Suprema & 10.28 & 5.09 & 1.39 & 1.5 \\
$50.8 \mathrm{~mm}$ GM-2 Hard Screw & 8.14 & 6.07 & 2.59 & 2.0 \\
\hline $25.4 \mathrm{~mm}$ Nominal & 5.42 & 4.74 & 1.26 & N/A \\
25.4 mm Suprema & 6.81 & 5.28 & 1.75 & 1.0 \\
$25.4 \mathrm{~mm}$ Polaris Flex Clamp & 8.78 & 6.73 & 2.49 & 1.0 \\
$25.4 \mathrm{~mm}$ Polaris Flex Spring & 15.20 & 10.76 & 4.37 & 2.5 \\
\hline
\end{tabular}

\subsection{Varying Torque on a Flat}

The minimum hold experiments showed that it is often possible to find a torque value for which the optic is securely held without introducing unacceptable amounts of wavefront error. However, to properly understand the effect a mount has on the wavefront quality of an optic, it is important to see how the deformation evolves as a function of torque. As such, the $50.0 \mathrm{~mm}$ flat mirror was tested under varying set screw torques with the Suprema mount to get a representative idea of what happens at different loads. The Suprema was chosen since it is the mount used for many fold mirrors in HCST.

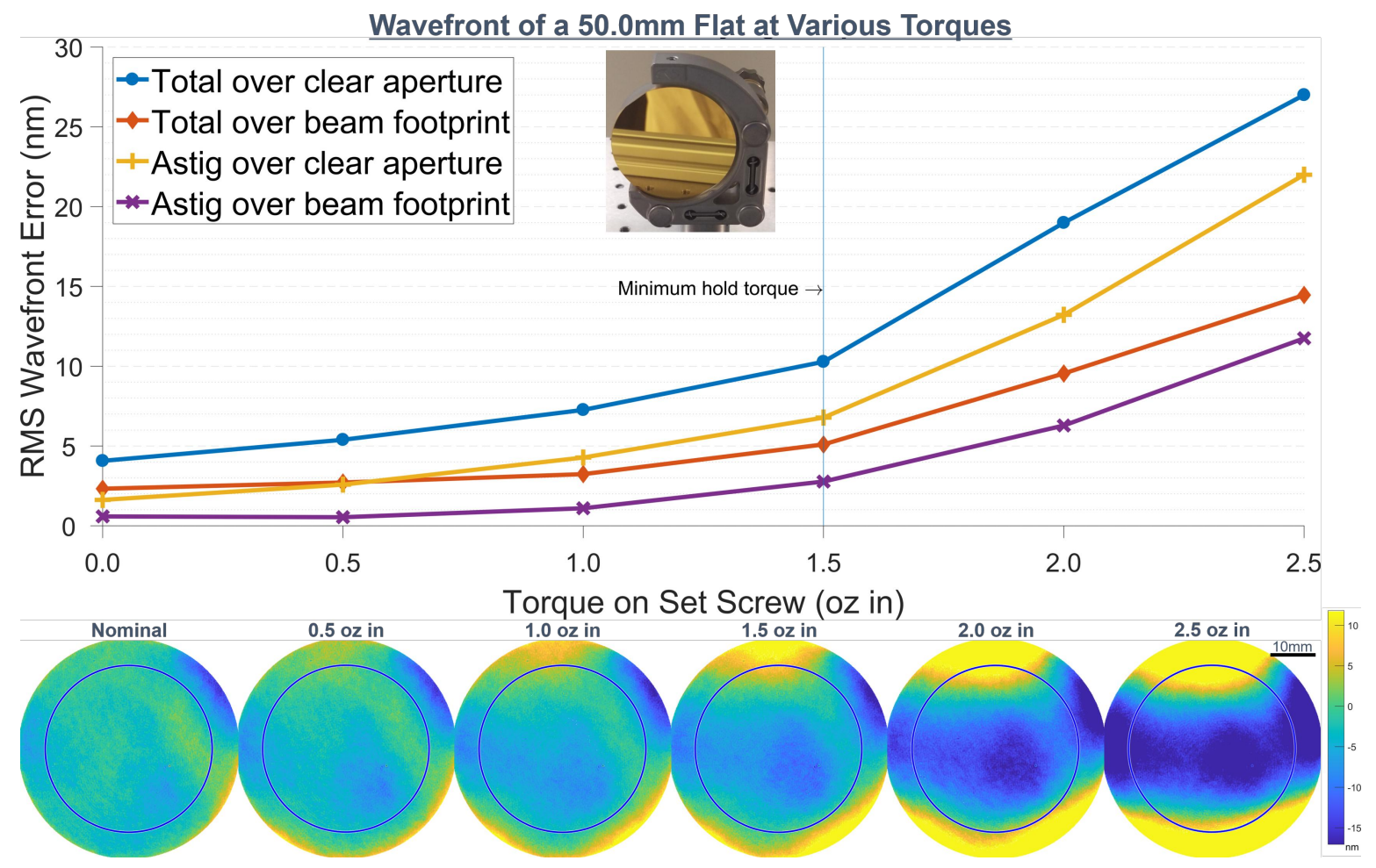

Figure 5. Effect of torque on a $50.0 \mathrm{~mm}$ Flat mirror in a Newport $50.8 \mathrm{~mm}$ Suprema mount, shown at the top of the graph. The graph displays several RMS error values as a function of torque, including the total RMS wavefront error over an $80 \%$ region (beam footprint) as well as the RMS astigmatism. The wavefront map at each torque is shown underneath.

Figure 5 shows the results of this test. One feature to note is that the red curve in the graph, the RMS wavefront error within the central $80 \%$ region of the mirror, has a plateau from 0 to $1 \mathrm{oz}$-in. Meanwhile, the blue 
curve, the RMS over the clear aperture, constantly increases from the start. This is likely due to the fact that the deformation in the mirror starts at the edges and works its way in towards the center so it is not immediately detectable in the region of interest. The wavefront maps show that effect more clearly as the mount's 3 contact points are visible in the 1.0 oz-in map and continue to grow until they dominate the surface in the last map. This leads to the intuitive conclusion that oversizing the optic relative to the beam size is better since the mounting effects will be less prominent within the region of interest. However, there is also a trade-off since larger optics deform more easily, as shown in Sec. 3.1, so the optic will need to be thicker as well.

The plateau in the red curve is also significant since it means that there is a range of torques for which the wavefront error is acceptable. Comparing this region with the results from Sec. 3.1 shows that the minimal holding torque occurred just after the plateau, as shown by the vertical line in Fig. 5. The same was true for most of the mounts tested. Beyond that plateau, the deformation started to grow rapidly and non-linearly so care must be given to not exceeding the acceptable region. Furthermore, the torques shown in Fig. 5 are very small. In fact, 1 ounce-inch is less than 7.1 millinewton-meters so it is very easy to over-tighten the set screw in a mount and significantly warp the optic without realizing it. This is why a new tuning and alignment procedure, detailed in Sec. 4, was developed for KPIC and HCST.

Finally, Fig. 5 shows that a large part of the wavefront error introduced by the mount was astigmatism. Figure 6 highlights this effect and shows that the dominating aberrations introduced by the mount were astigmatism and defocus. These terms cannot be corrected for with the flat itself so, as stated in the next section, they can lead to alignment problems when they propagate to OAPs in the system. Thus, tuning the optic to ensure that the aberrations are minimized is key or else errors in flats can continue to accumulate.

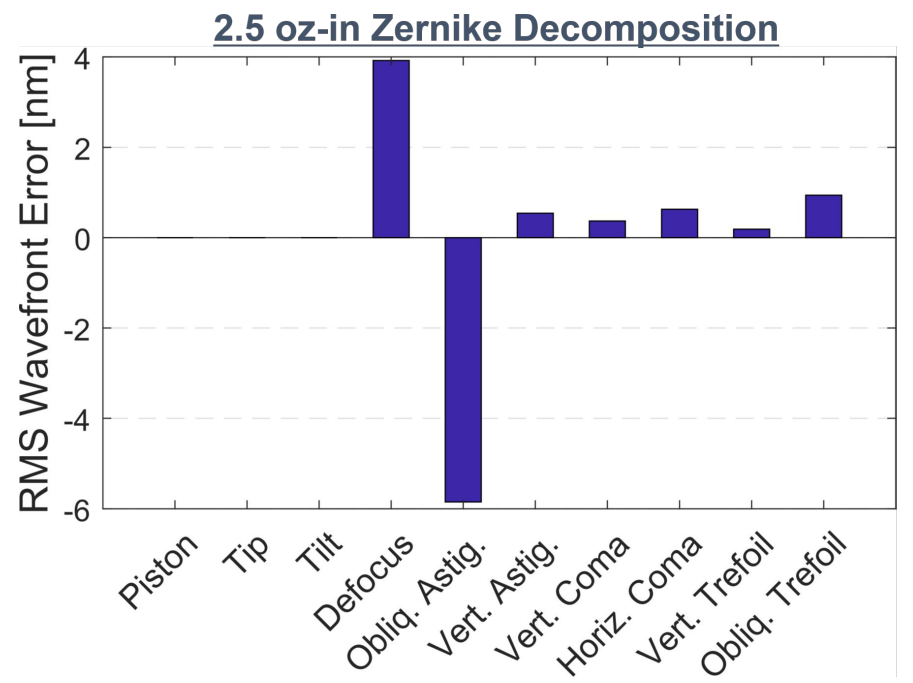

Figure 6. RMS contribution of the first 10 Zernike polynomials to the total wavefront error in the 2.5 oz-in test case for the Suprema mount. The first three terms are negligibly small since they come from alignment and were removed during analysis.

\subsection{Varying Torque on an OAP}

The varying torque test was repeated with one of the $50.0 \mathrm{~mm}$ OAPs from HCST to check if the same effects were seen with the non-flat mirrors and custom OAP mounts which have 2 flexure arms to hold the optic. The procedure described in Sec. 2 for testing OAPs was used with one slight modification: the set screw for the side flexure was tightened just enough to make contact with the OAP while the top screw was varied. This was done to be completely certain that the OAP would not turn during the tests but it did introduce a slight astigmatism to the nominal measurement in the data set. The results of this test are shown in Fig. 7.

The graph of RMS wavefront error for the OAP has the same key features seen with the flat in Fig. 5: there is a plateau in all the curves, astigmatism is the primary aberration present as the torque increases, and the slopes 


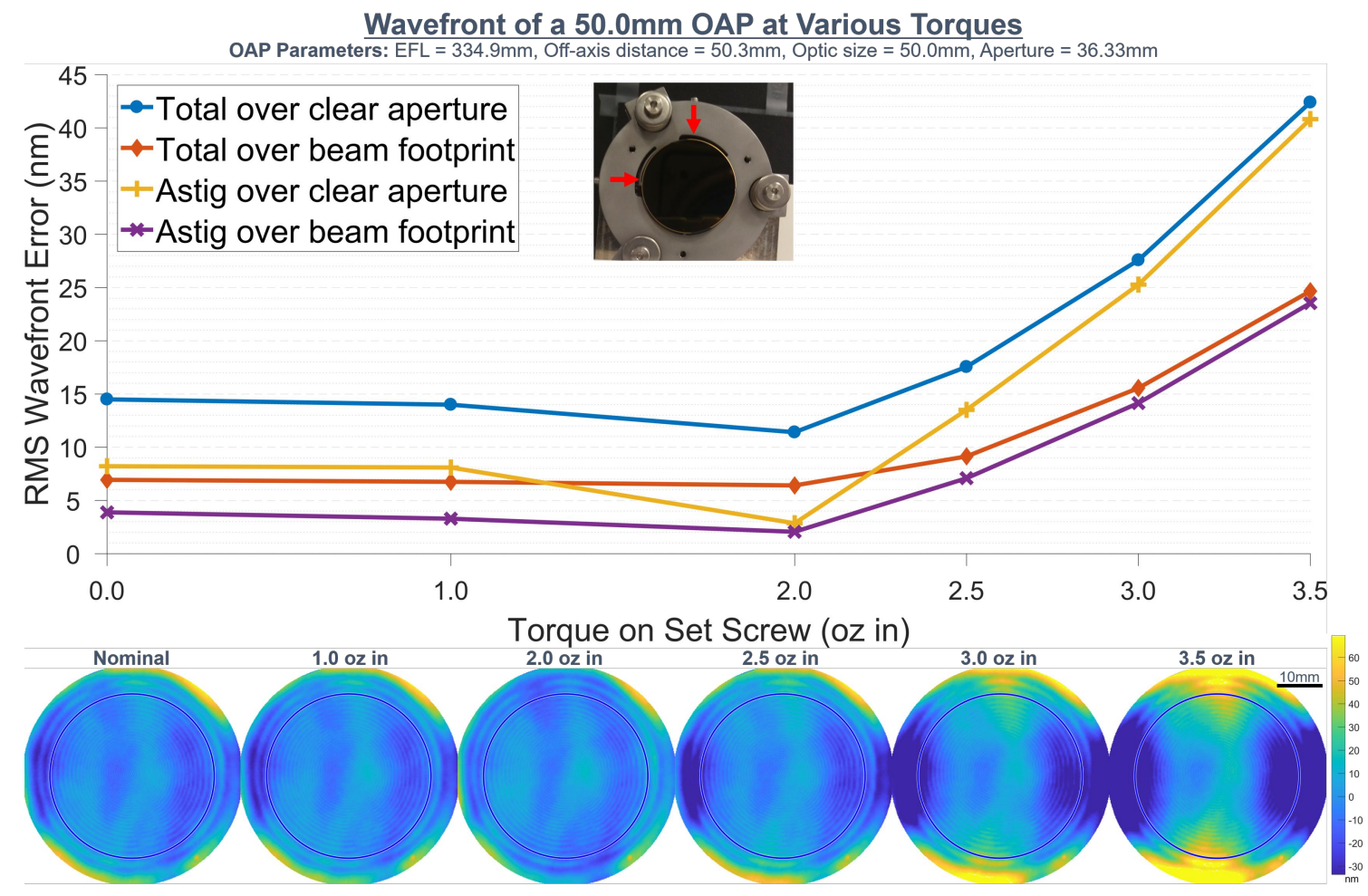

Figure 7. Effect of increasing torque on the wavefront of a $50.0 \mathrm{~mm}$ OAP. The mount used here was a custom OAP mount from HCST, shown at the top of the graph; note the two flexure arms marked by red arrows. The graph displays several RMS error values as a function of torque. As before, the wavefront maps have the same color bar.

of the curves for the full optic are steeper than for the $80 \%$ region. The main difference between the graph for the OAP and that of the flat is that the astigmatism over the clear aperture seems to decrease temporarily, with a minimum at $2.0 \mathrm{oz}$-in, before starting to increase. However, this effect is likely caused by the side set screw in the OAP test. The side screw introduced some astigmatism which was then cancelled by the top screw as its torque was increased but as the top screw continued to increase, the astigmatism from the top screw surpassed that caused by the side screw and thus the overall astigmatism started to grow again. As such, both the OAP and the flat show the same behavior with respect to torque despite the fact that the flat made direct contact with the set screw while the OAP was isolated from its set screws by flexure arms.

The fact that the primary aberrations are astigmatism and defocus is both a good and a bad thing for alignment. It is good since it means that the dominating mount-induced errors can be removed by shifting or angling the OAPs in the system. However, it is bad because removing it causes the beam to deviate from the designed path which can be problematic for systems with fixed locations for the optics. For example, HCST uses baseplates which constrain the location of all the mounts such that large deviations from the prescribed optical-path cause the beam to land close to the edge of the downstream mirrors. This puts the beam in the region of the optics where the mounting stresses are strongest. Thus, the tuning process was critical to the alignment since it ensured that the mount-induced aberrations were minimized and hence that the beam path remained true to the design.

\section{ALIGNMENT CONSIDERATIONS}

Even the best mounts will still introduce some aberrations. These can be significant if not taken care of but they can also be minimized easily. One way to reduce the deformation in an optic would be to simply torque the set screws in the mount as little as possible but a better way is to quantitatively assess the optic as it is placed 
and secured in the mount. This can be done with any wavefront sensor but for HCST and KPIC, this was done using a Zygo interferometer as described in Sec. 2.

Each optic was individually placed and tuned in front of the Zygo. The screws were backed off to start and a nominal measurement was taken as a reference for the wavefront error from the optic itself. Then a Tohnichi ATG18Z torque gauge, capable of measuring up to 18 ounce-inches, was used to determine the torque at which the optic started to deform since, as shown in the previous section, deformation starts just before the minimal holding torque. The optic was then tapped to check if it was secure. If it was not, the torque was increased slightly and the optic was tapped again. This was repeated until a secure hold was achieved. The final wavefront was then measured to ensure it was at an acceptable level. For all the HCST optics, an RMS wavefront error of less than $10 \mathrm{~nm}$ was achievable. The process was repeated for each optic even though some of them were identical since it was found that there was some variability within the same mount models which could cause unnecessary deformation. This variability is likely due to small differences like the set screw threads and the amount of lubrication present on each screw. Regardless, independently tuning each one ensured they all met the wavefront error requirement.
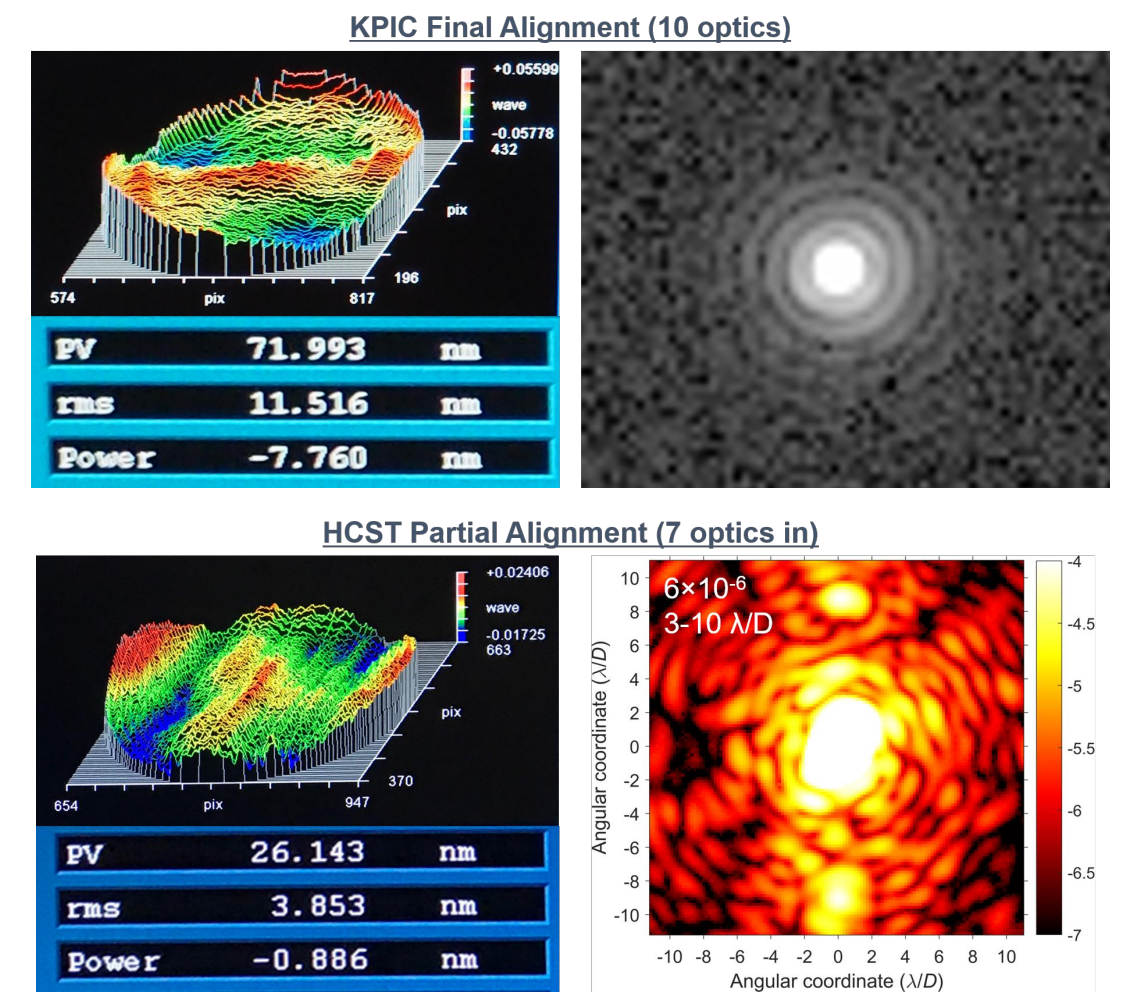

Figure 8. Wavefront quality of KPIC (top) with all 10 optics aligned and HCST (bottom) with the first 7 optics aligned. The values are reported in surface height, ie. half the total wavefront. The first-light PSF of both systems is also shown but the HCST PSF has the coronagraph in place. Note the KPIC PSF was taken at 1550 nm while the HCST PSF was collected at $800 \mathrm{~nm}$.

Both KPIC and HCST were also aligned with the Zygo after tuning was done. This allowed us to monitor and minimize the wavefront through the system as each optic was added. Thus, the mount-induced aberrations left after the tuning process, seen in Figs. 3 and 4, were removed through alignment. Optimizing the wavefront at each step provided extremely low wavefront error in both systems. KPIC, which has 10 optics, was aligned to a net wavefront error of about $25 \mathrm{~nm}$ RMS, while HCST, with 17 optics, was aligned to a net wavefront error of under $30 \mathrm{~nm}$ RMS. Figure 8 shows the wavefront quality of these systems as well as the first-light PSF of each. Note that a mean contrast of $6 \times 10^{-6}$ was achieved in the mid spatial frequencies on HCST with the vortex coronagraph and the DM flattened. This matched the prediction that maintaining less than $10 \mathrm{~nm}$ RMS per 
optic would provide a contrast better than $10^{-5}$.

The tuning and the alignment of KPIC and HCST provided some useful feedback on the design of both systems. It was found that the modular nature of HCST was particularly useful for alignment. HCST uses baseplates to ensure that the optics are placed in the correct position and to enable easy removal of sub-sections of the system. ${ }^{6}$ During alignment it was found that despite the tuning process, minimizing the alignment errors required that some of the optics be moved slightly from their nominal position. The baseplate design prevented the mounts within any given plate from moving with respect to each other but adjacent plates could be translated relative to one another to fully remove the wavefront aberrations. These motions were generally small but beyond the tolerance included in the plate holes for the mounts. The original HCST design had a single, large baseplate for the first 17 optics and made it impossible to fully minimize the errors since there was not enough slack in the mount locations. The new design only placed four to five mounts per plate and provided more options for optimizing the wavefront error. As such, the modular nature was useful for alignment as well as experimental flexibility.

\section{CONCLUSION}

We have shown that special consideration should be given to the mounts in high-contrast imaging systems since they can introduce significant wavefront errors. Some of the mounts presented can easily quadruple the RMS wavefront error within an $80 \%$ region of a mirror with as little as 3.5 ounce-inches of torque on the set screw. During the initial alignment of HCST, it was determined that these deformations were enough to change the beam path within the system as alignment adjustments were made to remove the mount-induced astigmatism and defocus. Tuning the torque on each optic individually minimizes these aberrations and while it is impossible to completely remove them and have the optic securely held, in most cases it is possible to reduce the errors to under $10 \mathrm{~nm}$ RMS. We have also shown that the mount-induced aberrations propagate from the edges of the mirror towards the center with increasing torque so oversizing the optic with respect to the beam beyond the $80 \%$ presented here further reduces the impact of the mount on the wavefront quality. These results were determined from a specific set of mirrors with specific material properties and dimensions but similar results can be expected with other mirrors as well. Additionally, though the torque values presented are unique to the mounts and optics tested and should not be taken as guides for other mounts, the procedures can be carried on to other testbeds. Taking these finding into consideration during the design and applying the procedures during alignment could greatly increase the overall wavefront quality and performance of future high contrast imaging systems.

\section{ACKNOWLEDGMENTS}

The authors would like to acknowledge the financial support of the Heising-Simons foundation. G. Ruane is supported by an NSF Astronomy and Astrophysics Postdoctoral Fellowship under award AST-1602444. This work was also supported by the Exoplanet Exploration Program (ExEP), Jet Propulsion Laboratory, California Institute of Technology, under contract to NASA.

\section{REFERENCES}

[1] Lawson, P. R., Belikov, R., Cash, W., Clampin, M., Glassman, T., Guyon, O., Kasdin, N. J., Kern, B. D., Lyon, R., Mawet, D., Moody, D., Samuele, R., Serabyn, E., Sirbu, D., and Trauger, J., "Survey of experimental results in high-contrast imaging for future exoplanet missions," Proc.SPIE 8864, $8864-8864-8$ (2013).

[2] Trauger, J., Moody, D., Gordon, B., Krist, J., and Mawet, D., "Complex apodization lyot coronagraphy for the direct imaging of exoplanet systems: design, fabrication, and laboratory demonstration," Proc.SPIE 8442, 8442 - 8442 - 13 (2012).

[3] Delorme, J., Mawet, D., Jovanovic, N., Wallace, J., Bartos, R. D., Wizinowich, P. L., Bond, C. Z., Cetre, S., Lilley, S., Jacobson, S., Echeverri, D., and Fitzgerald, M., "First version of the fiber injection unit for the keck planet imager and characterizer, paper 10702-77," Proc.SPIE, SPIE (2018). 
[4] Cai, W., Kim, D. W., Zhou, P., Parks, R. E., and Burge, J. H., "Interferometer calibration using the random ball test," in [International Optical Design Conference and Optical Fabrication and Testing], International Optical Design Conference and Optical Fabrication and Testing, OMA7, Optical Society of America (2010).

[5] Parks, R., Evans, C., and Shao, L., "Calibration of interferometer transmission spheres," OSA Technical Digest Series 12, 80-83 (01 1998).

[6] Jovanovic, N., Ruane, G., Echeverri, D., Delorme, J., Mawet, D., Fucik, J., Wallace, J., Coker, C., Delacroix, A., Levraud, N., Sayson, J. L., Wang, J., Riddle, R., and Millar-Blanchaer, M. A., "High-contrast spectroscopy testbed for segmentedtelescopes: instrument overview and development progress, paper 10702-159," Proc.SPIE , SPIE (2018). 